\title{
Fluoroscope guided epidural needle insertioin in midthoracic region: clinical evaluation of Nagaro's method
}

\author{
Won Joong Kim, Tae Hwa Kim, Hwa Yong Shin, Hyun Kang, Chong Wha Baek, Yong Hun Jung, \\ Young Cheol Woo, Jin Yun Kim, Gill Hoi Koo, and Sun Gyoo Park \\ Department of Anesthesiology and Pain Medicine, College of Medicine, Chung-Ang University, Seoul, Korea
}

Background: In the midthoracic region, a fluroscope guided epidural block has been proposed by using a pedicle as a landmark to show the height of the interlaminar space (Nagaro's method). However, clinical implication of this method was not fully evaluated. We studied the clinical usefulness of a fluoroscope guided thoracic epidural block in the midthoracic region.

Methods: Twenty four patients were scheduled to receive an epidural block at the T6-7 intervertebral space. The patients were placed in the prone position. The needle entry point was located at the junction between midline of the pedicle paralleled to the midline of the T7 vertebral body (VB) and the lower border of T7 VB on anteroposterior view of the fluoroscope. The needle touched and walked up the lamina, and the interlaminar space (ILS) was sought near the midline of the VB at the height of the pedicle.

Results: The authors could not insert an epidural needle at T6-7 ILS in two patients and it was instead inserted at T5-6 ILS. However, other patients showed easy insertion at T6-7 ILS. The mean inward and upward angulations were $25^{\circ}$ and $55^{\circ}$ respectively. The mean actual depth and calculated depth from skin to thoracic epidural space were 5.1 $\mathrm{cm}$ and $6.1 \mathrm{~cm}$ respectively. Significant correlation between actual needle depth and body weight, podendal index ( $\mathrm{kg} /$ $\mathrm{m})$ or calculated needle depth was noted.

Conclusions: The fluorposcope guided epidural block by Nagaro's method was useful in the midthoracic region. However, further study for the caudal shift of needle entry point may be needed. (Korean J Anesthesiol 2012; 62: 441-447)

Key Words: Analgesia, Epidural, Fluoroscopy, Thoracic vertebrae.

Received: July 13, 2011. Revised: September 7, 2011. Accepted: September 21, 2011.

Corresponding author: Young Cheol Woo, M.D., Ph.D., Department of Anesthesiology and Pain Medicine, College of Medicine, Chung-Ang University, 224-1, Heukseok-1dong, Dongjak-gu, Seoul 156-756, Korea. Tel: 82-2-6299-2584, Fax: 82-2-6299-2585, E-mail: wj12@naver.com This is a thesis for a master's degree.

(c) This is an open-access article distributed under the terms of the Creative Commons Attribution Non-Commercial License (http:// creativecommons.org/licenses/by-nc/3.0/), which permits unrestricted non-commercial use, distribution, and reproduction in any medium, provided the original work is properly cited. 


\section{Introduction}

A significant number of patients experience marked pulmonary insufficiency after a thoracotomy, due to severe postoperative pain. This post-thoracotomy pain, if not properly controlled, leads to difficulty taking deep breaths and coughing, which are associated with atelectasis, hypoxia, pneumonia, or respiratory distress - conditions interfering with postoperative recovery and lengthening the patients' hospital stays [1]. Management of post-thoracotomy pain, therefore, is an important matter, and studies have been conducted for its investigation. Postthoracotomy pain treatment methods using intramuscular or intravascular injection of opioid drugs, as well as intercostal nerve blocks using local anesthetics, are among the techniques studied. The most effective method reported is continuous infusion of local anesthetic into the epidural space $[2,3]$.

A thoracic epidural block is a relatively more difficult procedure than procedures used in other regions, because the spinous process of the thoracic vertebra is longer than that of the lumbar vertebra, and the area through which the needle can approach the epidural space is relatively smaller due to an acute angle and larger distance between the skin and the epidural space. The midthoracic region (T5-8) is the most difficult area when performing a block. Successful placement of the Tuohy needle is known to depend on the patient's physical conditions, anatomic changes in vertebrae, and the anesthesiologist's proficiency, among other factors, resulting in a failure rate of approximately $10 \%$ of the Tuohy needle placements attempted blindly by even the most skilled clinicians $[4,5]$. Another consideration to be made for a thoracic epidural block is the risk of serious neurological complications such as spinal cord injury, possibly resulting from the smaller epidural space in the thoracic region compared to that in the lumbar region, and from the presence of the spinal cord in the direction of the needle's path. Given these difficulties, it is essential that anesthesiologists perform the epidural block in the safest way possible [6].

Until recently, few reliable reports have been presented on fluoroscopice guided epidural blocks performed in the midthoracic region. Nagaro et al. [7] attempted such an epidural block using a paramedian approach, in which, after confirming that the interlaminar space (ILS) is located at the height of the vertebral pedicle in all thoracic vertebrae, they placed the entry point of the needle at the site where the inferior endplate of the targeted vertebral body (VB) meets, in the lateral position, the line parallel to the vertebral column that passes through the midline of the pedicle. They then attempt to locate the ILS from around the midline of the VB at the height of the pedicle [7]. Their study, however, involved only five patients on whom an epidural block was performed in the midthoracic region
(T6-7). The results of a study involving such a small number of subjects should be interpreted with caution when performing an epidural block in the midthoracic area.

The aim of this study was to evaluate the viability of a C-arm guided epidural block performed in the midthoracic region using a Tuohy needle. The procedure was based on the method reported by Nagaro et al. [7].

\section{Materials and Methods}

Among the patients scheduled for a thoracotomy with general anesthesia using inhalation anesthetics, 24 patients interested in receiving postoperative pain management were enrolled in the study. Each patient's written consent was collected after obtaining approval from this hospital's institutional review board. All candidates were Class 1 or 2 patients according to the ASA physical status classification system; patients for whom the use of the Tuohy needle is prohibited, who had abused drugs within two weeks prior to the thoracic epidural block, or were pregnant were excluded from the study. The subjects' age, height, weight, podendal index (PI), sex (sex ratio), and other demographic information are listed in Table 1.

The subjects arrived in the operating room unpremedicated and were connected to the ECG, blood pressure meter, and pulse oximeter. They were offered an explanation on how to use the patient controlled epidural analgesia (PCEA). The Tuohy needle (Epidural-set ${ }^{\circledR}$, Insung Medical, Korea) was placed by an anesthesiologist, using the procedure described as follows. The patients, in an awake state, were instructed to assume the prone position and to place a $10 \mathrm{~cm}$ deep pillow under their chest. The pillow was adjusted in such a way that the highest point on the patients' back, which would serve as the needle's skin entry point, was highlighted. The patients were also instructed to place both of their arms above their head to ensure that the C-arm fluoroscope would not be inhibited. A paramedian approach, in which the needle punctures the skin on the contralateral side from the operation site, was used.

The C-arm guidance AP view was adjusted such that the T7 spinous process would be situated at the midline of the $\mathrm{VB}$ and the $\mathrm{T} 7 \mathrm{VB}$ would form a square. The site for needle insertion was then cleansed and sterilized with Betadine ${ }^{\circledR}$ solution and

Table 1. Demographic Data

$\begin{array}{lc}\text { Age }(\mathrm{yr}) & 59.4 \pm 10.7(24-77) \\ \text { Gender }(\mathrm{M} / \mathrm{F}) & 16 / 6 \\ \text { Height }(\mathrm{cm}) & 162.8 \pm 8.4(151-176) \\ \text { Weight }(\mathrm{kg}) & 62.3 \pm 6.8(52-75) \\ \text { PI }(\mathrm{kg} / \mathrm{m}) & 38.3 \pm 3.8(29.5-45.5)\end{array}$

Values are mean \pm SD (minimum-maximum), PI (podendal index) is weight divided by height. 
covered with sterile cloths. Using a 25-gauge needle, a local infiltration of $2 \%$ lidocaine was performed at the entry point, and a 17-gauge Tuohy needle was advanced through the skin. The needle entry point was located at the junction between midline of the pedicle paralleled to the midline of T7 VB and the lower border of T7 VB on AP view of the fluoroscope (Fig. 1). The Tuohy needle was pushed into the skin to form an angle of approximately $10^{\circ}$ medially until it reached the lamina of the vertebra. The needle was then withdrawn slightly and advanced further medially as well as cephalad until the needle tip was situated in the midline at the height of the pedicle. Assuming the needle would enter the ILS if it did not touch the bone, the needle was further advanced - slowly and using the hanging drop method - until it arrived in the epidural space, at which point the number of times the needle touched the lamina was counted. A contrast agent (Omnipaque ${ }^{\circledR}$, GE Healthcare, 300 $\mathrm{mgI} / \mathrm{ml}$ ) was administered, with the location of the epidural space confirmed in AP as well as lateral views.

Based on the AP views, the angle formed between the inserted needle and the VB midline was measured and then denoted as the inward angulation (IA) (Fig. 2). From the lateral view, the angle between the needle and the line extending to the vertebral body's posterior border was measured and denoted as the upward angulation (UA) (Fig. 3). To investigate the relationships between the locations of the Tuohy needle tip, pedicle, and VB, the shortest distance between the Tuohy needle tip and both pedicles' lower borders and the vertebral body's lower border was measured in the AP view, the height

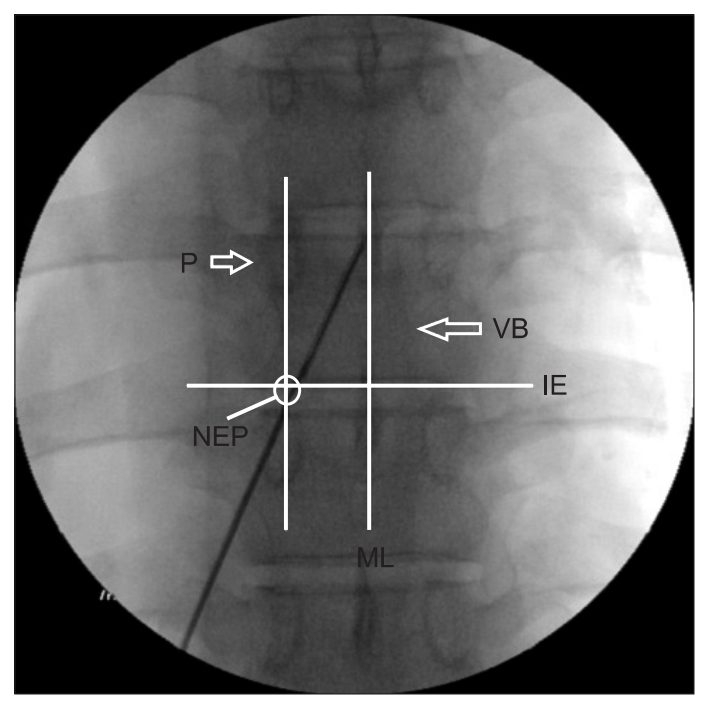

Fig. 1. This figure shows the interlaminar space, pedicle and vertebral body on AP radiograph. The needle entry point is the junction between the midline of the pedicle and the lower border of vertebral body. P: pedicle, VB: vertebral body, ML: midline, IE: inferior endplate of vertebral body, NEP: needle entry point. of the pedicle and that of the VB were measured, and the ratios (e/a, f/c) were obtained (Fig. 4). The actual needle depth extending from the skin entry point to the epidural space was measured, and the correlation coefficient between the actual depth and patients' weight, height, PI, and the calculated depth determined by using the pre-surgical computed tomography (CT) scans was obtained. To obtain the calculated depth, the line linking the epidural space between T6-7 and the spinous process was used (Distance B) (Fig. 5A), and a calculation was performed using the IA and UA, along with the Pythagorean

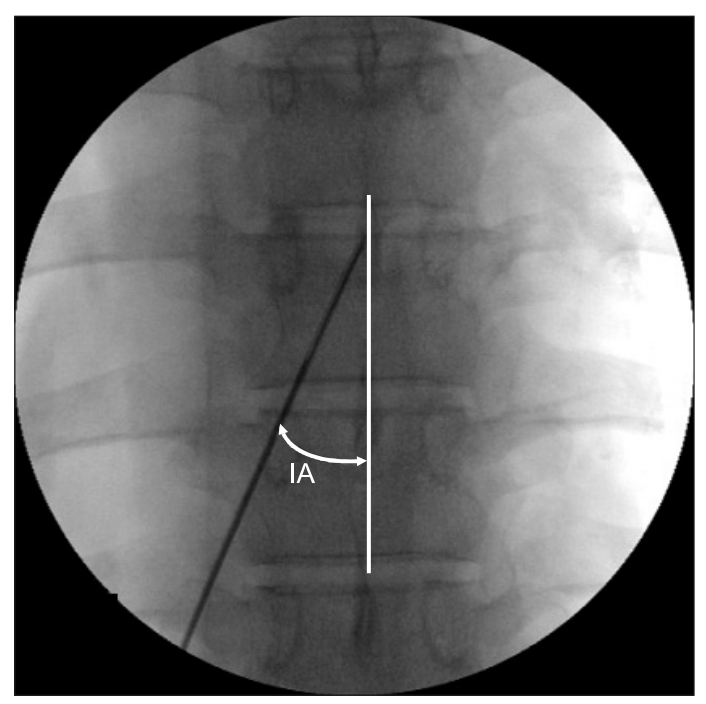

Fig. 2. The figure shows the inward angulation, which represents the angle of the needle to the line of the spinous process on AP view. IA: inward angulation.

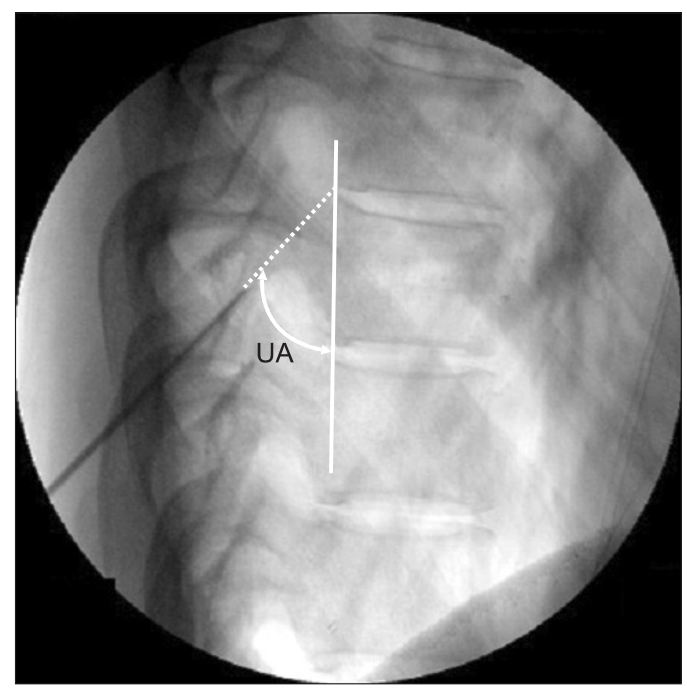

Fig. 3. The figure shows the upward angulation, which represents the angle of the needle to the line made by the posterior border of the vertebral body (white line) on lateral view. UA: upward angulation. 
trigonometric identity (i.e., calculated depth $=\mathrm{B} / \cos [\mathrm{IA}]^{\circ} \times \cos$ $[90-\mathrm{UA}]^{\circ}$ ) (Fig. 5B)

All measurements were presented as mean and standard deviation. The Shapiro-Wilk test was used for correlation normality tests; additionally, Pearson's correlation was used. Statistical analysis was conducted using SPSS Version 15.0 (SPSS Inc., USA). A P value of $<0.05$ was considered statistically significant.

\section{Results}

In 22 of the 24 subjects, the Tuohy needle was inserted into the T6-7 ILS without difficulty. In the other two subjects, the needle was inserted into the T5-6 ILS instead. These two subjects were excluded from all statistical evaluations.

Out of the 22 patients, three subjects represented cases in

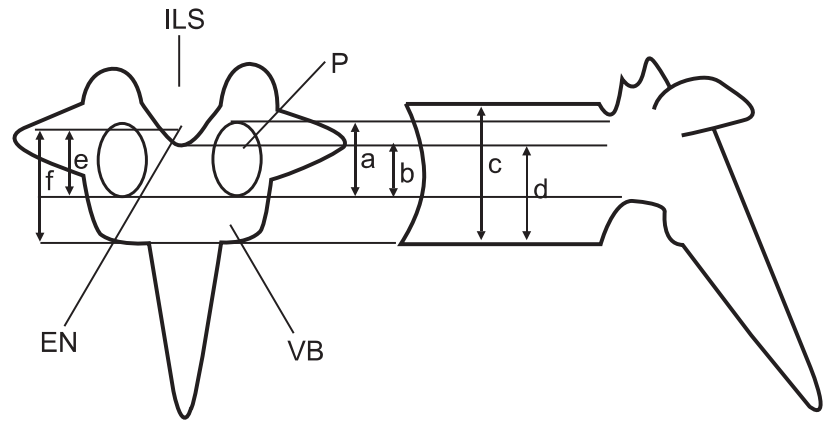

Fig. 4. A schematic representation that depicts the projection of a thoracic vertebra (T7) and shows the height of the interlaminar space and epidural needle tip relative to the pedicle and vertebral body. This figure is referred to in Nagaro's study, but ILS cannot be determined on AP view in this study. P: pedicle, VB: vertebral body, ILS: interlaminar space, EN: epidural needle, a: length of pedicle, $\mathrm{b}$ : distance from the lower border of the pedicle to the interlaminar space, c: length of vertebral body, d: distance from the lower border of vertebral body to the interlaminar space, e: distance from the lower border of the pedicle to the epidural needle tip, f: distance from the lower border of vertebral body to epidural needle tip. which the needle touched the lamina three times, and four patients represented cases in which the lamina was touched by the needle twice. In the remaining 15 patients, the needle came into contact with the bone once. The IA was $24.8^{\circ} \pm 5.5^{\circ}$ (minimum value: $12^{\circ}$, maximum value: $34^{\circ}$ ), and the UA was $54.5^{\circ} \pm 7.1^{\circ}$ (minimum value: $36^{\circ}$, maximum value: $70^{\circ}$ ). In most patients, the tip of the needle was found to be situated between the upper half of the height of the T7 pedicle and the inferior border of the $\mathrm{T} 6 \mathrm{VB}$, on the side of the puncture from the midline. Out of the 22 patients, the needle tip was found to be situated above the T6 VB inferior border in one patient (5\%); situated between the T6 VB lower border and the superior

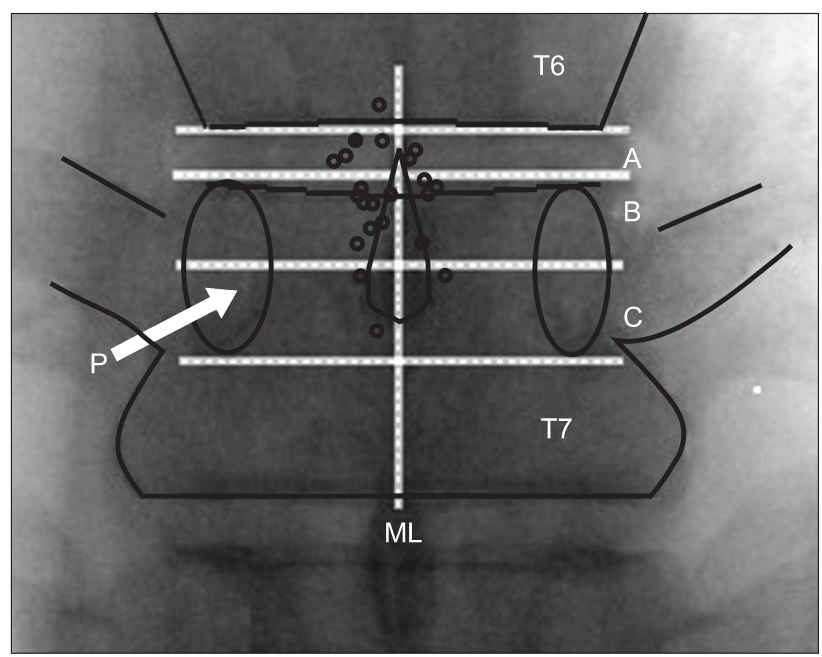

Fig. 6. The figure shows the location of the epidural needle tips (hollow circle and solid circle) with fluoroscope guided epidural block. The hollow square refers to the ipsilateral side of the needle entry point, but the solid square is the contralateral side. Most needle tips are located at the space A and B. A: space between T6 lower border and upper border of the T7 pedicle, B: space between upper border and horizontal midline of the T7 pedicle, C: space between horizontal midline and lower border of the T7 pedicle, ML: midline, P: pedicle.
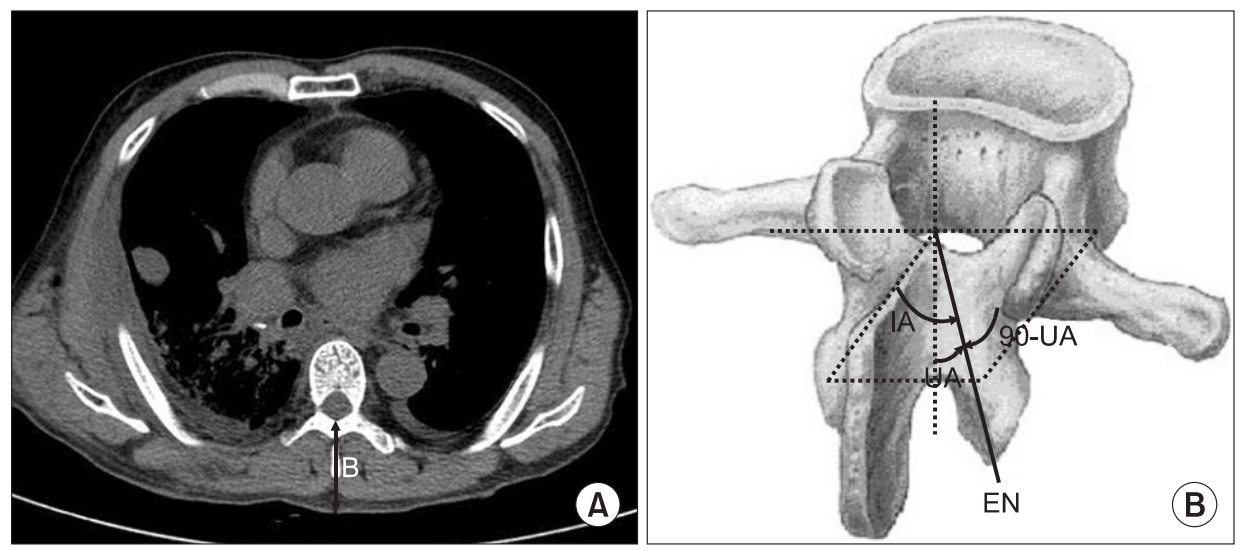

Fig. 5. (A) The midthoracic computed tomography film (transverse plane). B: The distance from the epidural space to skin at the T6-7. (B) Principle of Pythagorean triangle trigonometry. Estimated depth $=\mathrm{B} / \cos (\mathrm{IA})^{\circ} \times \cos$ $(90-\mathrm{UA})^{\circ} ; \mathrm{B}=$ Distance from the epidural space to skin at the T6-7 (CT transverse plane). IA: Inward anglulation (sagittal plane), UA: upward anglulation (axial plane), EN: epidural needle with paramedian approach. 
border of the $\mathrm{T} 7$ pedicle in six patients (27\%); between the superior border and the horizontal midline of the T7 pedicle in 12 patients (55\%); between the horizontal midline and the inferior border of the T7 pedicle in three patients (13\%); and beyond the vertical midline of the VB in one patient (5\%) (Fig. 6). The ratio between the pedicle length (a) and the distance between the inferior border of the pedicle and the needle tip (e) (e/a) was $0.76 \pm 0.32$ (minimum value: 0.13 , maximum value: 1.2), and the ratio between the height of the vertebral body (c) and the distance between the inferior border of the vertebral body and the needle tip (f) (f/c) was $0.98 \pm 0.13$ (minimum value: 0.75 , maximum value: 1.35 ) (Fig. 4).

The actual depth from the skin entry point to the epidural space was $5.1 \mathrm{~cm} \pm 0.6 \mathrm{~cm}$ (minimum value: $4.1 \mathrm{~cm}$, maximum value: $7 \mathrm{~cm}$ ), and the correlation coefficients between the epidural space's actual depth and the patients' weight and PI were 0.737 and 0.783 , respectively; these values were statistically significant $(\mathrm{P}<0.01)$. The distance between the epidural space and the skin measured in the CT scans was $4.3 \mathrm{~cm} \pm 0.6 \mathrm{~cm}$ (minimum value: $3.6 \mathrm{~cm}$, maximum value: $6.2 \mathrm{~cm}$ ). The distance obtained by trigonometry (i.e., the calculated depth) was $6.1 \mathrm{~cm}$ $\pm 1.0 \mathrm{~cm}$. The calculated depth was longer than the actual depth by $0.9 \mathrm{~cm} \pm 0.8 \mathrm{~cm}$; the correlation coefficient between the two values was 0.563 , which was significant $(\mathrm{P}=0.014)$.

\section{Discussion}

This study was conducted to evaluate the viability and clinical applicability of a fluoroscope guided thoracic epidural block, based on the method reported by Nagaro et al. [7].

In this study, the inward angulation (IA) was $24.8^{\circ} \pm 5.5^{\circ}$, which differed from the figure of $35^{\circ} \pm 8^{\circ}$ reported by Nagaro et al. [7]. We assumed this difference could be attributed to the location of the needle tip. In the study by Nagaro et al., the needle tips were placed above the vertical midline of the VB in all five patients [7], whereas in this study the needle tip was placed, in most cases, slightly laterally from the vertical midline, on the ipsilateral side of the skin entry point. The upward angulation (UA) in this study, $54.5^{\circ} \pm 7.1^{\circ}$, differed slightly from the figure reported by Nagaro et al. [7], $56^{\circ} \pm 11^{\circ}$. The UA is critical in determining the distance between the skin and the epidural space. In a study by Yang et al. on the depth of the thoracic epidural space measured from the skin, the distance between the skin and the T6-7 epidural space, measured in the sitting position via a paramedian approach, was $6.16 \mathrm{~cm}$ $\pm 1.25 \mathrm{~cm}$ [8], while the distance between the skin entry point and the thoracic epidural space in this study was $5.1 \mathrm{~cm} \pm 0.6$ $\mathrm{cm}$. In other words, considering that the patients' demographic information (e.g., height, weight, sex) in this study was similar to the information in the study by Yang et al., the approach used in this study appears to have resulted in a larger UA than that obtained by a blind method [8]. In fact, the lateral views showed that in this study there were more cases in which the angle between the needle and the posterior border of the vertebral body was larger than the angle between the midline of the upper and lower ILS and the posterior border of the vertebral body.

Based on the method adopted by Nagaro et al., the correlations between the distance from the skin to the epidural space and the patients' weight and PI, respectively, were examined. In the epidural block using a median approach, a significant correlation was reported between the patients' weight and PI, respectively, and the distance between the skin and the epidural space $[9,10]$. Located between the skin and the epidural space are several structures, including the subcutaneous tissue, the supraspinous ligament, the interspinous ligament, and the ligamentum flavum. The reported correlation between the patients' weight and the depth of the epidural space can be explained by the fact that the subcutaneous tissue is the structure with the highest variability among patients [11]. In a paramedian approach, the angle created between the needle and the skin surface could vary; in addition, deviation of the measurements could increase because the needle tip could likely be placed in ILS on the contralateral side of the entry point. Shim et al. [12] reported that there were no correlations between the patients' height, weight and PI, the angle between the skin surface and the needle in the thoracic vertebrae, and the depth of the thoracic epidural space. In the study described here, however, the entry point was determined by fluoroscopy. Thus, the reference entry point was the same for all cases, as was the target location of the needle point. As a result, the IA and UA in this study were consistent to a certain degree, contributing to the significant correlations found between the patients' weight, the PI and the depth of needle insertion.

In this study, the correlation between the actual depth extending to the thoracic epidural space and the CT-guided calculated depth was also examined. The calculated depth was found to be larger than the actual depth, a finding consistent with the results reported by Kao et al. [13] in a study in which the depth was measured in the lower thoracic region (T10-11), yet contradictory to the results of Lee et al. [14], in whose study the actual depth in the midthoracic region (T7-8) was found to be larger than the calculated depth. The reason why the calculated depth was larger than the actual depth in our study is presumably that the position the patients were instructed to assume (i.e., the prone position, with support from a $10 \mathrm{~cm}$ deep pillow) had tightened the skin more than in the supine position where, as in a CT scan, the patients lie on their back, with their arms raised. This prone position, then, had ultimately caused the patients' subcutaneous tissue to receive more 
pressure and become more compressed, thus rendering the actual depth smaller when measured. Therefore, an important implication is derived: when advancing the Tuohy needle into the thoracic epidural space, the clinician should expect to find the space in a shallower spot than the calculated depth, and thus should suspect that any further advancing of the needle (i.e., all the way into the estimated depth) could carry the risk of damaging the spinal cord.

Nagaro et al. chose the pedicle as the needle's target reference site because they reasoned that they could reduce variability between patients due to the fact that the pedicle is associated with the least amount of anatomical variation and degenerative changes found in individual patients [15]. Using the skeletons of five cadavers, the researchers verified that the caudal edges of the ILS in all thoracic vertebrae, including the midthoracic region, and all lumbar vertebrae, were situated at the height of the pedicle, and that they were all situated at a height half that of the VB or above the VB. From fluoroscope guided AP views, the researchers then determined that the ratio between the length of the pedicle in T7 (a) and the distance between the pedicle's lower border and the ILS caudal edge (b) (b/a) was $0.6 \pm 0.1$. They also obtained the ratio between the length of the VB (c) and the distance between the VB inferior border and the ILS caudal edge (d) (d/c), which was found to be 0.7 \pm 0.1 (Fig. 4). In this study, we originally intended to verify the aforementioned findings in our patients, using a C-arm guide. However, we found it impossible to achieve a clear examination of the ILS caudal edge in the AP views due to the obstruction of fluoroscopy by the long spinous processes of the thoracic vertebrae, the acute angle formed by these, and the organs situated in the mediastinum. Therefore, we chose to calculate the abovementioned ratios using the needle tip placed in the epidural space. According to our calculations, the ratio between the pedicle length and the distance between the pedicle's inferior border and the needle tip was $0.76 \pm 0.33$, and the ratio between the VB length and the distance between the VB lower border and the needle tip was $0.98 \pm 0.13$. These figures were slightly larger than those obtained by Nagaro et al., presumably because the needle tip in our study was placed into the epidural space, slightly toward the cranial side rather than toward the ILS caudal edge.

In the majority of the 22 patients participating in this study, the needle tip was found to be placed somewhere between horizontal midline of the T7 pedicle and the T6 VB inferior border. The ratio between the pedicle length (a) and the distance between the pedicle's lower border and the needle tip (e) (e/a) was, on average, 0.76, indicating that in most patients, the interlaminar space began in the upper half part of the pedicle. However, since the minimum value was 0.13 , caution is required when the needle is pushed in toward the lamina; such a first trial could lead to a penetration into the ILS. The maximum value, on the other hand, was 1.2, indicating that in certain cases the ILS began above the lower border of the VB in the upper level along the thoracic vertebrae.

The approach adopted by Nagaro et al. appears to offer a clearer guideline on the needle entry point and target site than that suggested by the blind method. In their approach, the IA and UA, also appear to be more consistent. The use of fluoroscopy enables clinicians to approach the epidural space at a particular angle, as well as to confirm the placement of the needle inside the epidural space when assisted by the use of contrast agents. This technique helps prevent complications resulting from epidural blocks, such as spinal cord injury. In the study described here, insertion of the Tuohy needle into the desired site was unsuccessful in two patients. Nevertheless, the needle was introduced safely into the epidural space in the upper level of the thoracic vertebrae. As a result, a 100\% Tuohy needle insertion rate was accomplished.

Several features of our study differ from those reported by Nagaro et al. For example, we carried out needle insertion in the prone position, not the lateral position. Inserting a Tuohy needle in the prone position has several merits. First, the prone position helps clinicians obtain C-arm guidance AP and lateral views with greater ease. Second, it is easier for the patients to maintain the same position throughout the procedure, without moving; it is also easier for the clinician to secure his or her visual field. Third, when verifying the thoracic level via fluoroscopy, the prone position makes it easier to count the bones (i.e., counting from $\mathrm{T} 1$ in the caudal direction). Lastly, when inserting the needle into the skin, the prone position helps reduce what is known as the dimpling of the skin.

Based on the results of this study, we suggest two ways to complement the method adopted by Nagaro et al. First, shifting the skin entry point slightly in the caudal direction would likely help the clinician locate the thoracic epidural space, since their method employs a larger UA, whereby the needle tends to approach the ILS at an acute angle instead of approaching it parallel to the lamina, and as a result increases the likelihood of failing to locate the epidural space. In this study, our attempt to insert the needle into the ILS between T6-7 failed in two patients; the insertion was accomplished in the space between T5-6 instead. The failure is presumably attributable to a UA that was larger than appropriate, in which case the needle tends to touch the lamina and then penetrate into ILS of the upper-level vertebra rather than the intended space. If the insertion must be accomplished in the ILS between T6-7, the skin entry point should be shifted toward the caudal edge, thereby reducing the UA.

Second, achieving a greater flexion of the thoracic vertebrae would enable clinicians to locate the thoracic epidural space 
with greater ease. In fact, not only the UA of the needle in the midthoracic region but also the degree of thoracic flexion, as well as the presence of osteophytes, influence the anesthesiologists' attempts to locate the thoracic epidural space. While it is more difficult than increasing the flexion of either the lumbar spine or the cervical vertebrae, accomplishing a greater flexion of the thoracic vertebrae, such as by increasing the depth of pillow beyond $10 \mathrm{~cm}$, as used in this study, would facilitate the attempt to locate the thoracic epidural space. In one of the 22 patients in our study, the UA was found to be $70^{\circ}$, showing a considerable difference from the average UA of $55^{\circ}$. The reason why the needle could penetrate into the epidural space despite such a large difference in UA was presumably the height of the interlaminar space.

In conclusion, the epidural block performed in the midthoracic region as described by Nagaro et al. is a clinically easy and safe procedure. We recommend further research on an alternative method in which the needle is inserted from a skin entry point that is situated slightly toward the caudal edge.

\section{References}

1. De Cosmo G, Aceto P, Gualtieri E, Congedo E. Analgesia in thoracic surgery: review. Minerva Anesthesiol 2009; 75: 393-400.

2. Sawchuk CW, Ong B, Unruh HW, Horan TA, Greengrass R. Thoracic versus lumbar epidural fentanyl for postthoracotomy pain. Ann Thorac Surg 1993; 55: 1472-6.

3. Lomessy A, Magnin C, Viale JP, Motin J, Cohen R. Clinical advantages of fentanyl given epidurally for postoperative analgesia. Anesthesiology 1984; 61: 466-9.

4. Hood DD, Dewan DM. Anesthetic and obstetric outcome in morbidly obese parturients. Anesthesiology 1993; 79: 1210-8.

5. Konrad C, Schüpfer G, Wietlisbach M, Gerber H. Learning manual skills in anesthesiology: Is there a recommended number of cases for anesthetic procedures? Anesth Analg 1998; 86: 635-9.

6. Giebler RM, Scherer RU, Peters J. Incidence of neurologic complications related to thoracic epidural catheterization. Anesthesiology 1997; 86: 55-63.

7. Nagaro T, Yorozuya T, Kamei M, Kii N, Arai T, Abe S. Fluoroscopically guided epidural block in the thoracic and lumbar regions. Reg Anesth Pain Med 2006; 31: 409-16.

8. Yang MK, Kim DH, Kim KW. A depth of the Tuohy needle in thoracic epidural anesthesia. Korean J Anesthesiol 1994; 27: 778-781.

9. Han KR, Choi HR, Hyun HS, Kwak NK, Kim C. A clinical measure of the skin to cervical epidural space depth in the Korean adults. J Korean Pain Soc 1999; 12: 114-8.

10. Song ZH, Kang YS, Chun TW, Cho KH. Distance from skin in the lumbar epidural space in Korean adult male. Korean J Anesthesiol 1994; 27 : 1635-8.

11. Palmer SK, Abram SE, Maitra AM, von Colditz JH. Distance from the skin to the lumbar epidural space in an obstetric population. Anesth Analg 1983; 62: 944-6.

12. Shim JC, Lee ME, Kim DW. Predicting factors for the distance from skin to the epidural space with the paramedian epidural approach. J Korean Pain Soc 1996; 9: 349-53.

13. Kao MC, Tsai SK, Chang WK, Liu HT, Hsieh YC, Hu JS, et al. Prediction of the distance from skin to epidural space for low-thoracic epidural catheter insertion by computed tomography. Br J Anaesth 2004; 92: 271-3.

14. Lee SJ, Choi SH, Kim MS, Shin YS. Prediction of the distance from skin to midthoracic epidural space by computed tomography. Korean J Anesthesiol 2005; 48: 605-8.

15. Kricun ME. Imaging modalities in spinal disorder. Philadelphia, WB Saunders. 1988, pp 59-288. 